\title{
ANDROID APPLICATION TO EXTRACT THE STATISTICS OF AN HPC CLUSTER
}

\author{
S.Chakraborty, Miraz Nabi Azad, Sourav Sen, Pritomrit Bora \\ Aditya Singh, Bipal Das and Mohd.Tabeesh Noori \\ Department of Computer Engineering, NIT Silchar, Assam \\ ww. nits.ac.in
}

\begin{abstract}
As the number of HPC systems across the globe is booming at a rapid pace. The task of system administration is turning out to be tedious. Using a computer for every petty work can be cumbersome. In recent times there has been a paradigm shift from PC to mobile on a rapid scale. So a need has risen for such paradigm shift in system administration too. To solve this problem, an android application has been developed which allows the admin to monitor the system statistics remotely using his cellphone.
\end{abstract}

\section{KEYWORDS}

Android, Eclipse, Java, JSCH, SSH, IPMI.

\section{INTRODUCTION}

Android platform is the most promising and widely used operating system in recent times for smart phones and other hand held devices. So it is a good platform to develop a prototype application so that the system administrator of an HPC cluster can remotely login to the system and retrieve data regarding system health which will help him to take appropriate measures if needed without being physically present in vicinity of the system simply by using his or her cell phone.

\section{OBJECTIVES OF PROPOSED PROJECT}

- The application will act as a client and the administrator can connect to the ssh server by providing valid credentials.

- The administrator can use this application to analyze the statistics of the cluster by extracting information such as Memory usage, Hardware information, CPU and processing information, Disk space statistics and Network Packet statistics.

- This application also enables user to run IPMI command so that user can analyze information such as Power Status, Fan Sensors, Temperature Sensor and System Event Log.

- The application can also be used for visual representation of the extracted cluster information. 


\section{SCOPE OF THE PROJECT}

Many android applications with similar prototypes have been researched and developed but the existence of one single concrete application such as our application HPC Health is dubious. This application overcomes the problem of constant monitoring of the HPC cluster by enabling admin to access the system remotely and remain updated about the state of the system. As a new solution to develop such a system, this paper introduces the use of secure shell protocol using java.

\section{RESEARCH IN THIS PAPER}

The key research element in this paper is the introduction of a System Administration tool in mobile platform. For decades SSH has been used for the task of system monitoring through a PC or work station, but this paper shows us the possibility of doing same by using hand held devices or smart phones with the help of this application, since mobilization is the future. This paper also introduces tool for convenient System monitoring by providing visual aid such as plotting graph from the extracted information in real time which makes it more perspicuous and helps in quick decision making.

\section{ANDROID}

Android is a linux based operating system targeted for smart phones and hand held devices. The OS was developed by Android Inc and owned by Google since 2005.Tools and different API's for developing mobile application are provided by android SDK. Android offers a unified approach to application development for mobile devices which means developer need only develop for android and their application shall run on different devices powered by android.

\section{SYSTEM ARCHITECTURE}

Android OS is a stack of software components that consist of five sections and four main layers.

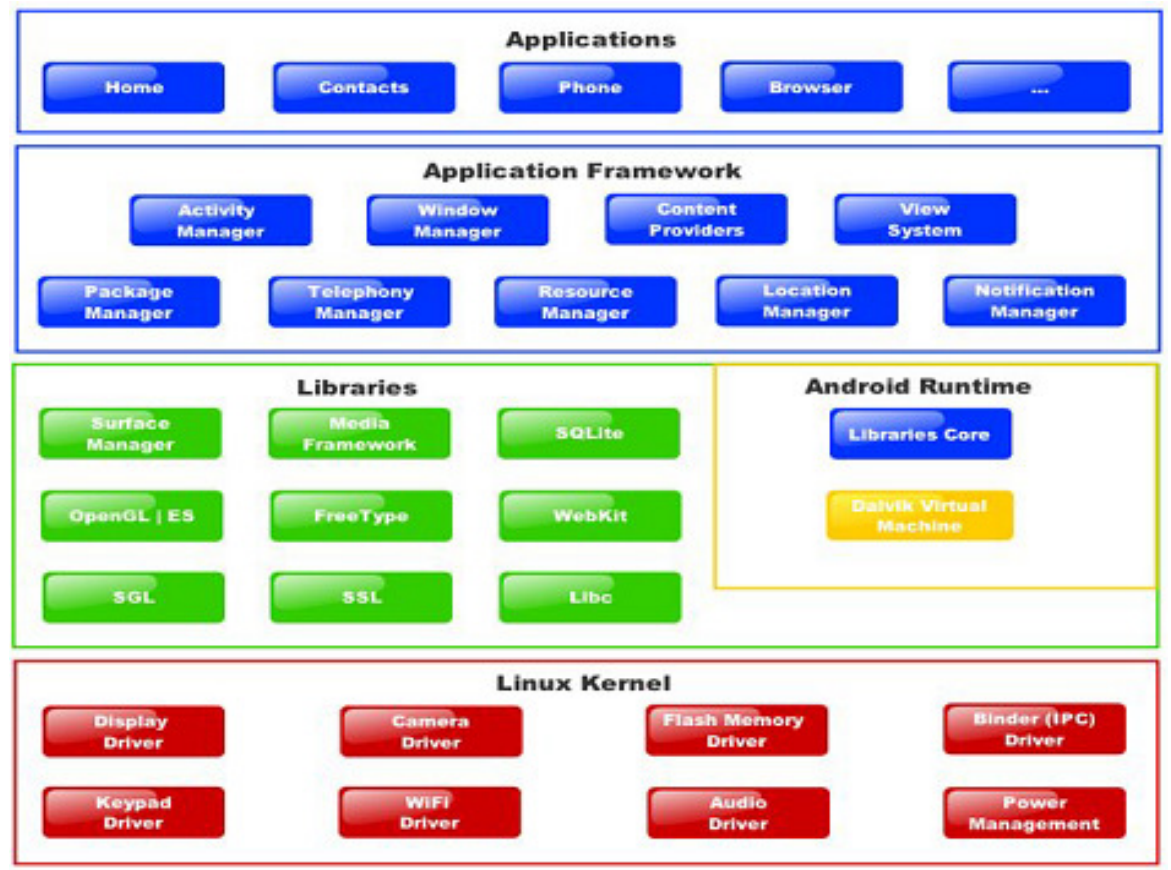

Fig 1. System architecture of Android 


\subsection{Android Application Development Environment}

Android applications are written in java and compiled into byte codes which will be converted to .dex file (Dalvik Executable File) using dx converter. This will further be complied into android package file (apk file) that can be installed to an android device.

Android application consists of one or more of these four components. The main building blocks are:

- Activity: It is the core component of Android Application. It is a user interface that dictates how the user interacts with smart phone screen.

- Services: It is designed to keep running in the background to perform tasks that perform long running operations.

- Content Providers: The content providers are used as an interface to data. Content provider helps maintain shared data between applications.

- Broadcast Receivers: These are broadcast announcements made by the system.

\section{TECHNOLOGIES USED}

- SSH AND Jsch: SSH is a unix based interface and protocol for securely getting access to a remote computer. It is widely used by network administrator to control web and other kind of servers remotely. It provides strong authentication and secure communication over in secure channel by encrypting transmitted data during SSH session.

- Jsch(Java Secure Channel): Jsch is a java implementation of SSH. It is a Java library which provides the implementation of SSH functionality.

- IPMI tool: IPMI(Intelligent Platform Management Interface) is a an open standard hardware management subsystem to communicate .IPMI is a single command line interface useful for managing IPMI enabled devices .It enables user to manage system hardware ,monitor system health, monitor and manage the system environment independent of the operating system.

\section{EXPERIMENTAL DESIGN}

- Firstly to connect the android device to the server, ssh connection was planned to be used to accomplish which jsch library was incorporated in the android environment.

- For communication between the client application and the server, input and output streams were created.

- The commands to be fired on the server were sent through the output stream and the results were channeled back to the device through the input stream.

- Graphs were plotted to check the performance of the HPC system using the data received. This was accomplished using the GraphView api for android. To give a real time view of the performance the graph data was refreshed every few milli seconds using threads. 


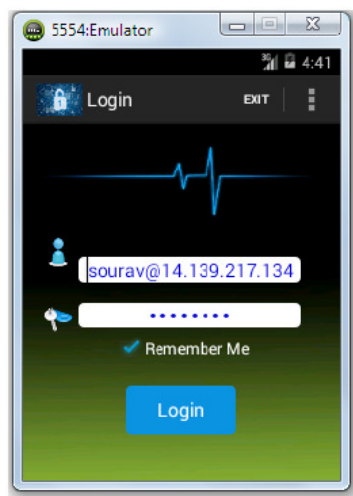

Fig 2. Login screen

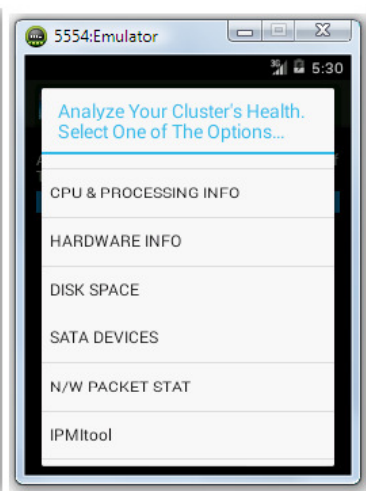

Fig 3. Menu screen

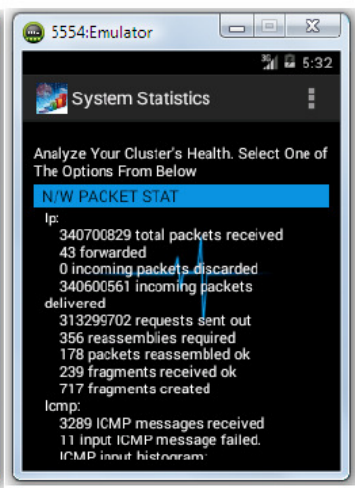

Fig 4. Output displayed

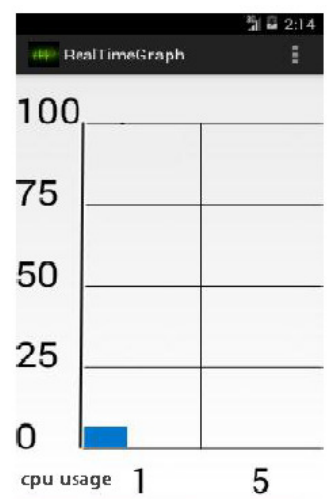

Fig 5. Percentage CPU usage graph

\section{OVERVIEW}

HPC Health will be a project consisting of Client and Server. The interaction between user and the system is depicted in the use case diagram given below.

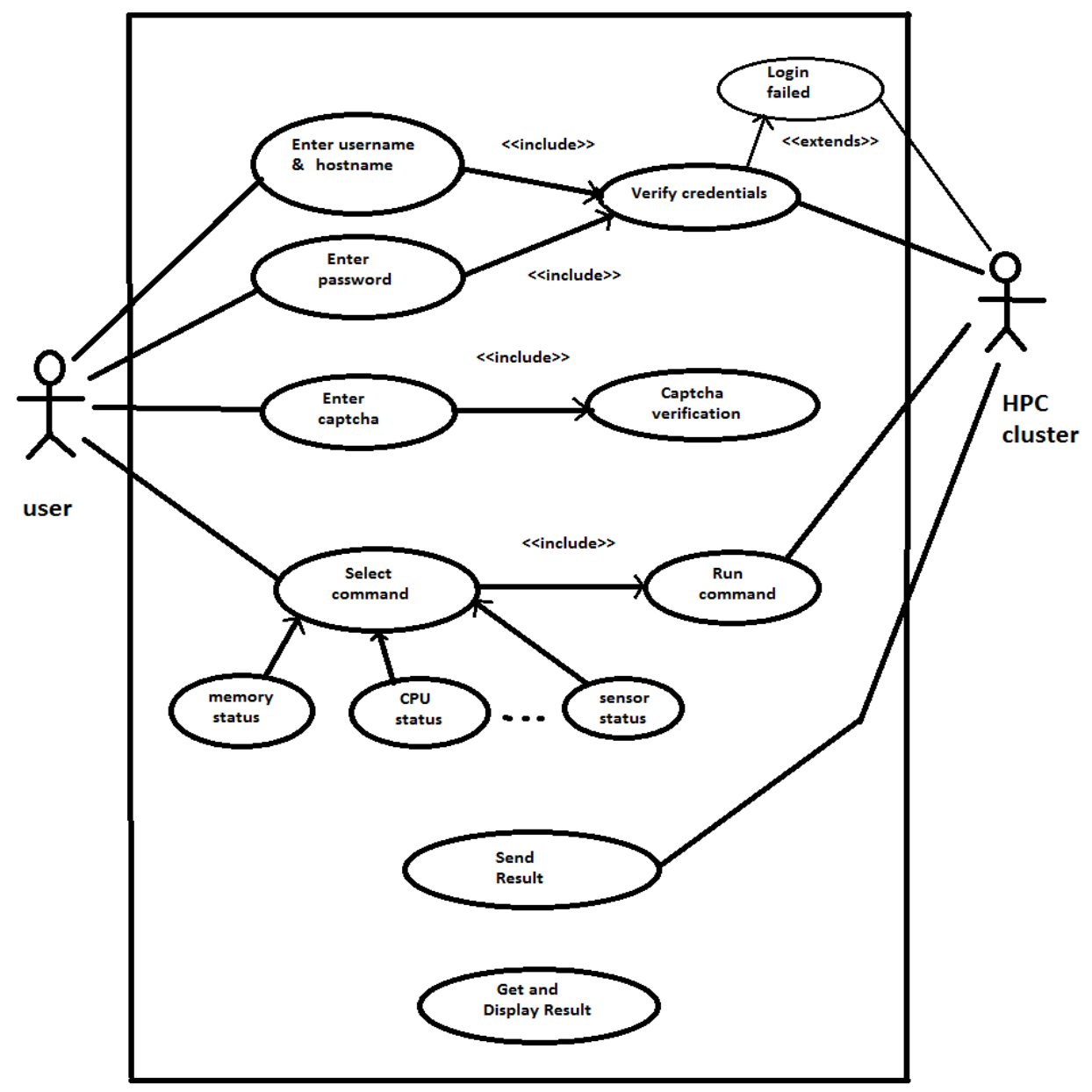

Fig 6. Use Case Diagram 
The order of events that take place while manoeuvring the application by the user is depicted below with a activity diagram.

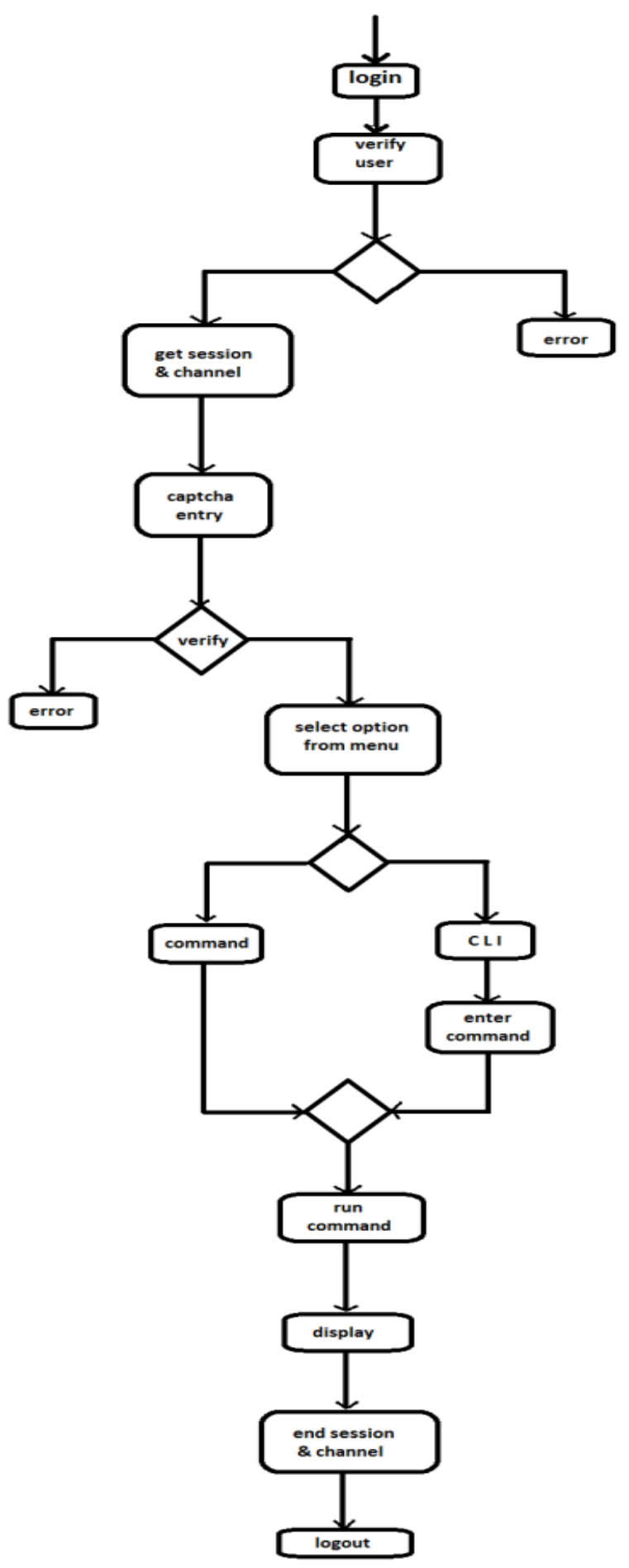

Fig7. Activity Diagram. 


\section{APPLICATION AND FUTURE SCOPE}

Due to the rapid expansion in technology, there is a need for fast computation which has led to a boom in the supercomputing industry. The increase in the number of HPC clusters has entailed for an advanced system administrating tool .In such a scenario our HPC Health application becomes a very competent tool as it provides features such as remote monitoring which makes the task of system administration very expedient.

There are a lot of features that could be incorporated into this application and we will attempt to target those in the coming months.

- The system administrator should be able to control the processes that are running in the system, start new ones and kill existing ones, if necessary.

- Our main aim will be to show all the processes in a drop down list so that user can select from them.

- We will try to incorporate more graphs in displaying the data so that it is more convenient for user to understand the state of the system.

\section{CONCLUSION}

This thesis project has covered mobile application development in Android platform. The basic components of android have been described along with the overview of the Android architecture. The outcome of this project has accomplished most of the goals we set at the start. This project was an opportunity to discover and explore new skills in the field of mobile application development. The final application can be useful to system administrators for keeping track of their systems (Cluster) remotely without disrupting the normal performance of the system.

\section{ACKNOWLEDGEMENTS}

We would like to extend our gratitude to Dr P.K. Sinha(Senior Director, HPC, CDAC), Mr Abhishek Das and the C-DAC members at NIT Silchar for their cordial support, valuable information and guidance provided by them, which helped us in moving ahead with this task through various stages. We are also very grateful for their cooperation during the period of our assignment.

\section{REFERENCES}

[1] Dr. Atsuhiko Yamanaka. Jcraft. JCraft, Inc. Web. 10 Jan. 2015.

[2] Jonas Gehring. Graph View. 16 Jan. 2015

[3] Ramzi N.Sansour, Nidal Kafri, Muath N.Sabha "A survey on mobile multimedia application development environment".

[4] Ms Loveena Lionel "Android As A Server Platform".

[5] Deepali Javale "Home Automation and Security System Using Android ADK"

[6] Andreas Sommer, Stephen Krusche "Evaluation of cross platform frameworks for mobile applications".

[7] Henning Heitkotter "Evaluating Cross Platform Development Approaches for Mobile Applications"

[8] Ifeanyi P. Egwutuoha, David Levy,Bran Selic Shiping Chen "A survey of fault tolerance mechanisms and checkpoint/restart implementations for high performance computing systems". 